$\S=-1$

\title{
Eye Controlled Robotic Chair with Night Vision Camera
}

\author{
G. Harish Babu' ${ }^{1 *}$ Dr. N. Venkatram² \\ ${ }^{1}$ Research Scholar, Koneru Lakshmaiah Education Foundation \\ ${ }^{2}$ Dean of Academics, Koneru Lakshmaiah Education Foundation \\ *E-mail: harish.sidhu12@gmail.com
}

\begin{abstract}
According to Census 2011, in India the population is $121 \mathrm{Cr}$, from the aggregate residents $2.68 \mathrm{Cr}$ individuals are impaired i.e 2.21 percent of the aggregate residents. From the $2.68 \mathrm{Cr}$ impaired individuals 20 percent of the people disability in movement. Our project is an attempt to make lives of the people suffering from this phenomenon simple. In this framework controlling of wheel chair is depends upon the movements of patient's Eye [1]. A camera is placed before the user, to catch the picture of the eye \& footmarks the locale of eye pupil. Camera used here is Night vision camera which works during night time and as well as daytime and will also work during low lightings. As per the position of eye pupil, wheel chair will be coordinated to move left, appropriate, forward and in reverse. The previous algorithms which are developed for this type of project doesn't support for the people with squint eye and supports only for the people with 2 perfect pupils, and those algorithms are works only in clear light conditions but this project supports for the people with squint eye as well as people with 2 perfect pupils and works during low lightings. The person can stop the chair by closing his eyes whenever he needs. Moreover, a ultrasonic sensor is ascended in front, so that it can recognize the snags and naturally stop the wheel chair. A GPS module is inserted which finds the location of the wheel chair. A GSM module is inserted which sends the messages in case of emergency situations. This project provides an option called voice module which is inserted and works based on the commands of the patients.
\end{abstract}

Keywords: Camera; GPS; GSM; ; Image Processing; Open Computer vision Library; Python; Raspberry Pi; Wheel chair

\section{Introduction}

In accordance with 2011 Census, in India, by the whole of $121 \mathrm{Cr}$ populace, around $2.68 \mathrm{Cr}$ people are 'impaired' i.e $2.21 \%$ of the aggregate populace. In the year 2011 in india persons $121.08 \mathrm{Cr}$, males $62.32 \mathrm{Cr}$, females $58.76 \mathrm{Cr}$ Persons $2.68 \mathrm{Cr}$, males $1.5 \mathrm{Cr}$, females as $1.18 \mathrm{Cr}$. Throughout the impaired populace $56 \%$ are males i.e $(1.5 \mathrm{Cr}) \quad \& 44 \%$ are females i.e $(1.18 \mathrm{Cr})$. In the aggregate populace, the female and male populace are $49 \%$ and $51 \%$ individually. Dominant part $(69 \%)$ of the crippled populace lived in country territories $(1.86 \mathrm{Cr}$ debilitated people in provincial zones \& $0.81 \mathrm{Cr}$ in metropolitan regions). On account of aggregate population additionally, $69 \%$ are from rustic territories while the staying $31 \%$ lived in urban regions.

In India, 20 percent of the impaired people possess infirmity in motion, 19percent are with incapacity in observing, and one more 19 percent are with inability in perception. 8 percent has various inabilities.

In association with the aggregate debilitated in the lifetime of (019)yrs, 20 percent retain incapacity in perception took after by 18 percent with inability in observing. 9 percent has different incapacities.

Amidst the crippled in the lifetime of (20-39)yrs, 22 percent possess infirmity in motion and 18 percent has incapacity in hearing. 6 percent has different inabilities.

$\square$ Through the crippled in the lifetime of (40-59)yrs, 23 percent retain handicap in movement and 19 percent has incapacity in observing. 5 percent has different inabilities.
Amidst the aged debilitated people, the incapacities in displacement (25 percent), in observing (25 percent) and perception (12 percent) are noticiable. 12 percent has various incapacities.

The below table gives the statistics of the paralysis in Asia for different countries.

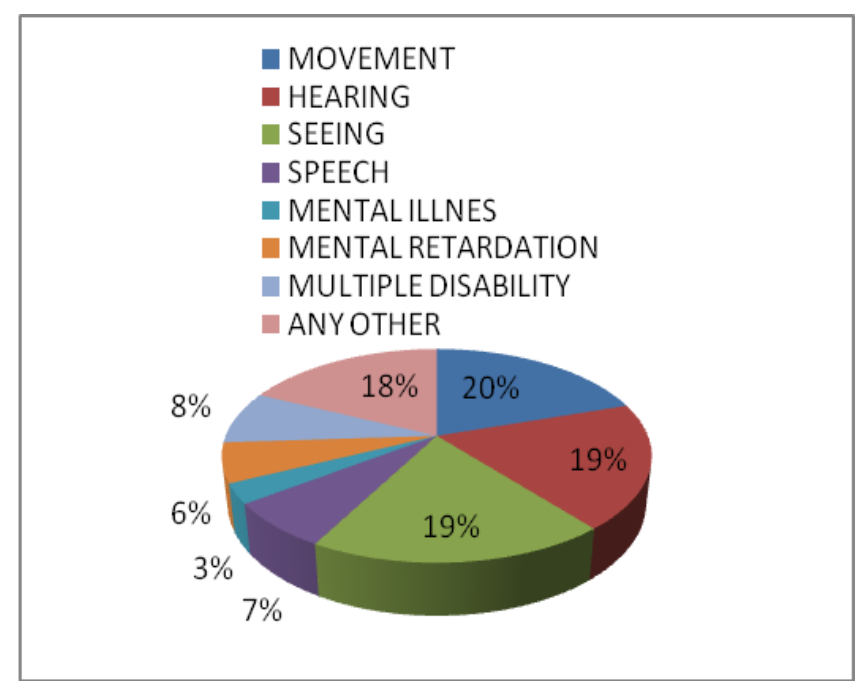

Fig 1: Disabled persons statistics in India as per 2011 census. 
Table 1: Units

\begin{tabular}{|l|l|l|}
\hline \multicolumn{3}{|c|}{ Paralysis in Asia (Extrapolated Statistics) } \\
\hline Bangladesh & $1,272,064$ & $141,340,4762$ \\
\hline Bhutan & 19,670 & $2,185,5692$ \\
\hline China & $11,689,628$ & $1,298,847,6242$ \\
\hline East Timor & 9,173 & $1,019,2522$ \\
\hline Hong Kong s.a.r. & 61,695 & $6,855,1253$ \\
\hline India & $9,585,635$ & $1,065,070,6072$ \\
\hline
\end{tabular}

Loss of motion (Paralysis) and Spinal Cord Injury (SPI) statistics from the Source of Christopher \& Dana Reeve Foundation Paralysis Resource Center USA. There are relatively 5.6 gobs individuals or 1.9 percent of the residents experience issues or failure to ploy atleast one upper or lower extremities.More than 1.2 million individuals has spinal string damage. The main sources of loss of motion is feat $-29 \%$, Spinal string damage $-23 \%$, various Sclerosis - 17\%, Cerebral Palsy - 7\%, Post Polio Ailment- 5\%, Other - $19 \%$. What's more the main sources of spinal string damage is On-the-tab mishap - 28\%, Motor conveyance mishaps $24 \%$, Sporting/Amusement mishaps - 16\%, Falls - 9\%, Other $23 \%$. This loss of motion influence yearly family pay of USA Approximately $59 \%$ of the population who got paralyzed family income is less than $\$ 25,000$, and approximately $63 \%$ of the population who are suffering from spinal cord injury report their family income of less than $\$ 25,000$.

This paralysis, Spinal card Injury patients, and disability with movement based persons their life is completely depend on others. It is very difficult to survive. Our research work focus on these problems to find a better solution to make their life simple with the help of Embedded like on board computer wheel chair system [2]. In last few years more and more wheel chair systems and models are available in our market. Some wheel chair systems are Joystick control based, some wheel chairs are gesture based control, Bluetooth based control [3] and Voice based controlled systems [4]. The wheel chair controlling with the help of Joystick is very difficult to control the wheel chairs lack these motor skills. It is difficult to guide in narrow openings such as doorway, without repeatedly colliding into the sides this type of system can be frustrating the persons. And gesture controlled wheel chair system it is not suitable for paralyzed persons. Voice controlled wheel chair system it will read the commands given by the patient and directs towards right, left, forward, reverse and stop. In any case a streaky system distracts the framework \& it will misguide the wheel chair and the problem is the patient should tell in a proper manner then only it will work otherwise it will waits this voice commands from the patient, in this voice controlled also having a drawbacks. Eye controlled wheel chair based system in this method based on the movement of the eye [8] the wheel chair will move right, left, forward and stop but this project will works only in high luminous conditions.

Now a day's most of the embedded system applications are perform based on image processing algorithms and it gives an accurate results. Image processing place a virtual role in most of the applications in the medical field as well as an autonomous robot control applications. In this paper we are control the wheel chair with eye movements. The Computer Vision tool box is used in this project for faces and eye detection. The algorithms and working of the project is explained in the system design model.

\section{System Design Model}

The figure 2 describes the hardware components of the entire system.

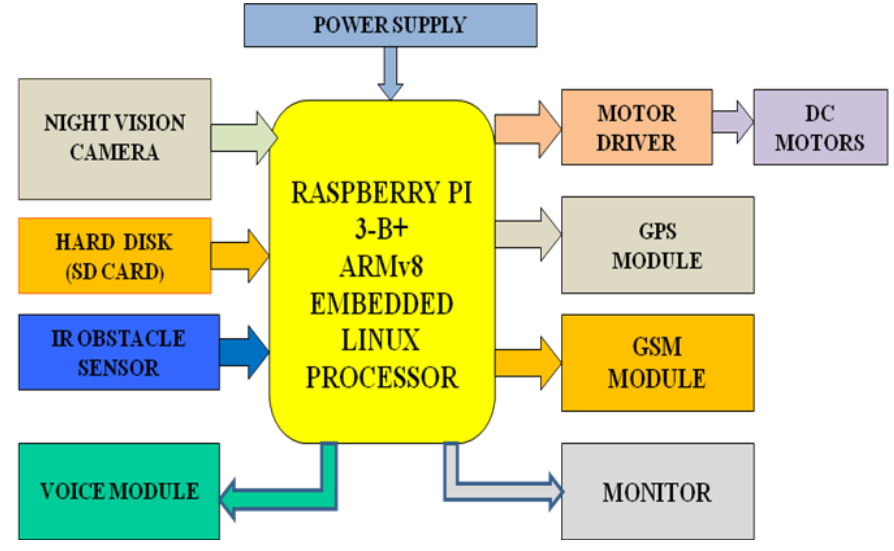

Fig 2: Block diagram of hardware system.

The main components of this system is power supply it is the basic requirement for any electronic equipment, night vision camera, dc motors, monitor, GPS module, GSM module, APR voice module, main brain of the entire system Raspberry Pi board and hard disk these are the basic requirements for this system. This system is cost effective and efficient for paralysis as well as disabled with movement problem persons. This system identifies face, eye and pupil location from the video image capturing [6] by the camera with minimum delay of time. The camera module is night vision camera it is work in both low luminous lightings as well as high luminous lightings. The hard disk size is $8 \mathrm{~GB}$ or $16 \mathrm{~GB}$ based on our application, the OS of the Raspberry Pi is "Raspbian " it is loaded into the hard disk and the application program is written in the python language. The raspberry pi board is a main component of this entire framework, the entire application program run in the Raspberry Pi. All the modules are connected and controlled by it. In this system all the control and command signals are generated by the raspberry pi.

Coming to the monitor section of this system the raspberry $\mathrm{Pi}$ board having a HDMI connector with the help of HDMA to VGA converter cable we can interface the general desktop monitors also. But the general desktop monitor requires $230 \mathrm{~V}$ AC supply. There are various monitors are available in our market with 4.3 inch HD LCD color display can also be interfaced with a voltage of $9 \mathrm{~V}$ $28 \mathrm{~V}$ DC but in our system we are using $16 * 2$ LCD is used it requires $5 \mathrm{~V} \mathrm{DC}$ it is sufficient for this system.

An ultrasonic sensor is utilized for hindrance recognition. With the help of this sensor we can find the distance of the object from the wheel chair. Based on the distance we can change the path of the wheel chair. This process leads to obstacle avoidance. Voice module is used to take the commands from the Raspberry pi. It produce small audio clips left, right, forward, backward and stop, it will help for the blind people to understand where they are moving In case of emergency situations, GPS module and GSM module is used to send a location and emergency alert to a caretaker. To track the person by Google maps [15][16]. The L293D is a double $\mathrm{H}$-Bridge engine driver, so with one IC we would interface be able to two DC engines (motors) which can be controlled in both clockwise \& against clockwise bearing [17-22].

Raspberry pi plays a major role in the hardware part. It processes the multiple images frames by frame efficiently. Specifications of raspberry pi Processor are Broad-com BCM2837 chip-set, Bluetooth 4.1, Wireless LAN $802.11 \mathrm{~b} / \mathrm{g} / \mathrm{n}$ and $1.2 \mathrm{GHz}$ Quad-Core ARM Cortex-A53. It has 24 GPIO pins and is connected to the motor driver, GSM, GPS modems. In this framework eye pupil location is very extremely testing to conquer this, a new image enhancing method is presented which depends on open CV [5] (open CV) library. In this pupil recognizable method we are utilizing a few calculations like Hough transform, Haar cascade, and edge recognition are accessible for various applications. 


\section{Methodology}

The guideline utilized in this framework is eye pupil identification and eye following in view of computer vision innovation. The location of the eye pupil is detected by using an algorithm based on image processing.

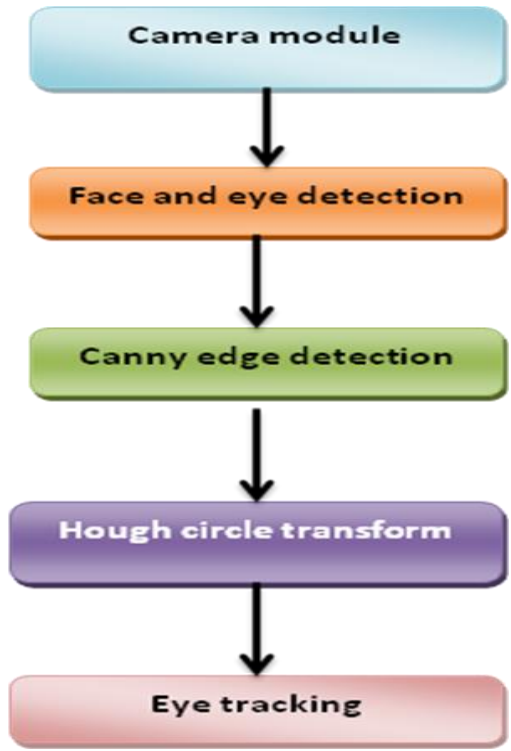

Fig 3: Working process for the system

There are a few phases present to discover the movement of the eye for example face recognition, eye identification, shading transformation, movement discovery. Night vision web cam captures images which are acquired by the system during the initial stage.

The algorithm is elaborated in a step-by-step manner.

Step 1: The web camera will capture the images.

Step 2: Used Haar cascade calculation for detection of face and eye.

Step 3: The task ahead is to recognise the eye pupil and mention its coordinates.

Step 4: Color conversion, filtering, edge recognition and hough transform are some of the image processing techniques which are used to reduce the size of image and increase the processing speed.

Step 5: To circle the pupil hough transform method is used.

Step 6: Coordinate system logic is used to calculate the farness between the eye circle centre point \& centre point. The separation will be changing as per the mobility of eye pupil. According to the movement of the pupil the user decides the direction in which he/she wants to travel.

The operation of the motors depends upon the movement of the pupil. For the right side of the motor to move the pupil should be moved to right. For the wheel chair to be moved in forward direction the pupil of the eye should be placed in the centre and both the motors start moving. Eye blinking logic is applied to start and halt the operations of the wheel chair. For the system to be completely stop the user has to close the eyes for 4 seconds. An emergency button is implanted on the chair which when pressed sends an emergency alert which consists of detailed location (latitude and longitude) of the user to the registered caretaker. The

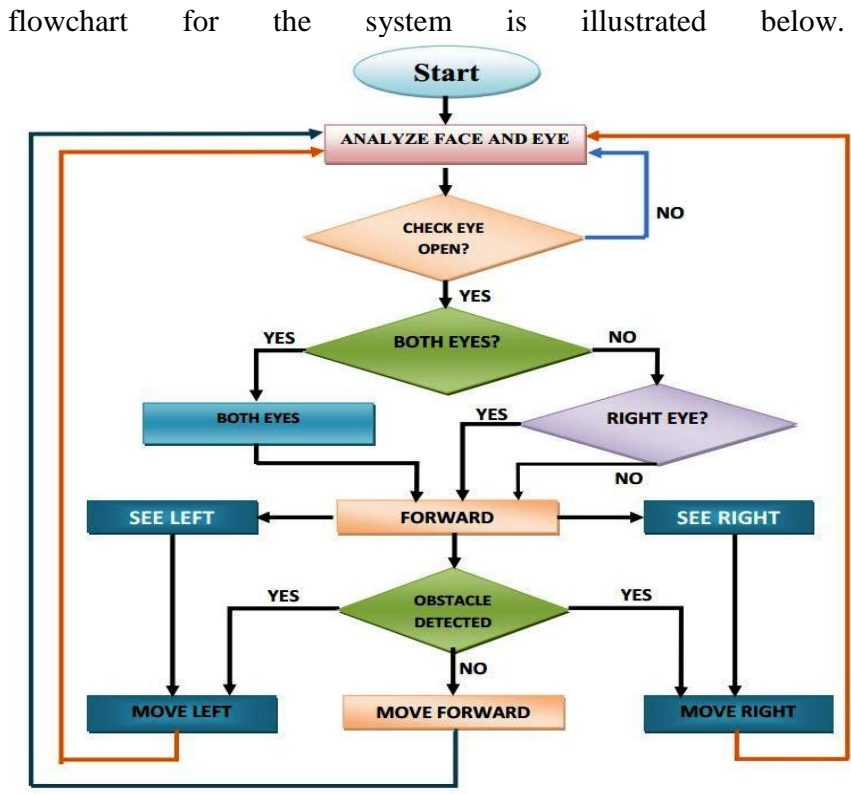

Fig 4: Flow chart of system working

Face and eyes are analyzed and checked whether the eyes are opened or closed. The eyes are open or close movementswill be decide the start and stop operation of the wheel chair [10]. If the eyes are opened it should be checked whether a single eye should be monitored or both the eyes for people having cross eyes. The single eye is checked to be right or left. If the eye is aligned in centre then the wheel chair moves forward. If the eye pupil is aligned to the right then the wheel chair moves right [14]. If the eye pupil is aligned to the left then the wheel chair moves left. If any obstacle is detected the wheel chair moves either left or right based on the movement of the pupil.

\section{Design Model of Framework}

This framework implementation depends on internal \& external environment. It helps the user to make life easier. This system is implemented with a raspberry pi3 board. The features of raspberry pi advanced board computer having 4 USB ports, HDMI, PWM, UART, 24 GPIO pins and ETHERNET port. It helps to connect internet [9] via wired or wireless connection to the external world. The hard disk capacity of Raspberry pi is up to $32 \mathrm{~GB}$ and the RAM is 512 MB. The night vision camera is mounted in a distance of 10 to $15 \mathrm{~cm}$ from the user's face. The camera is in fixed position. With the help of night vision camera we can capture the images and process in to the Raspberry Pi. With the use of system algorithm we can asset the exact pupil locale\& give the correct information to the board of raspberry pi. The board collects pupil location information \& it will give control signals to the motor driver IC. The motor driver circuit has two relays and connected with $9 \mathrm{~V}$ battery for rotating motors to the desired direction either left, right, forward or stop. In case of emergency the emergency switch is provided in this system to send an emergency message and location coordinates longitude, latitude by GPS module to the respective caretaker.

\subsection{Operating system installation steps in Raspberry $\mathbf{P i}$}

The name of the operating system used is "Raspbian jessie" [12] which is downloaded from the Internet with a .ISO format. The .ISO format is converted to bootable file format with the help of additional software known as "Win32 Disk Imager". The steps are to be followed to convert .ISO format into bootable format are shown in below.

Step 1: Install the Win32 Disk Imager and selects the path of the .ISO file and select the Bootable location. 


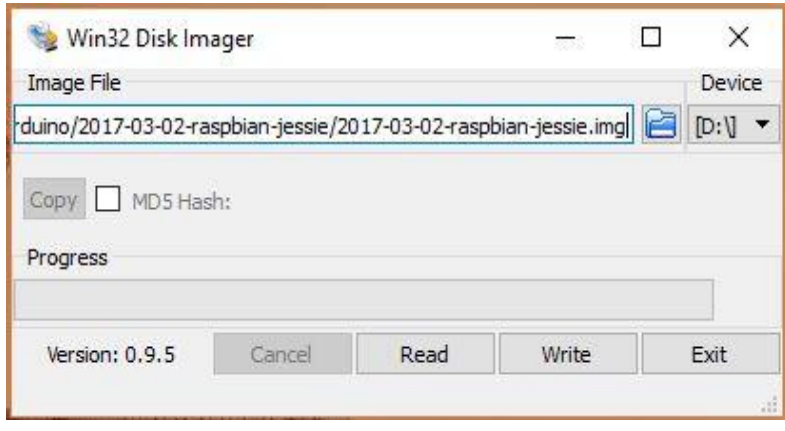

Step 2: click on Write option it will write the .ISO format into Bootable format.
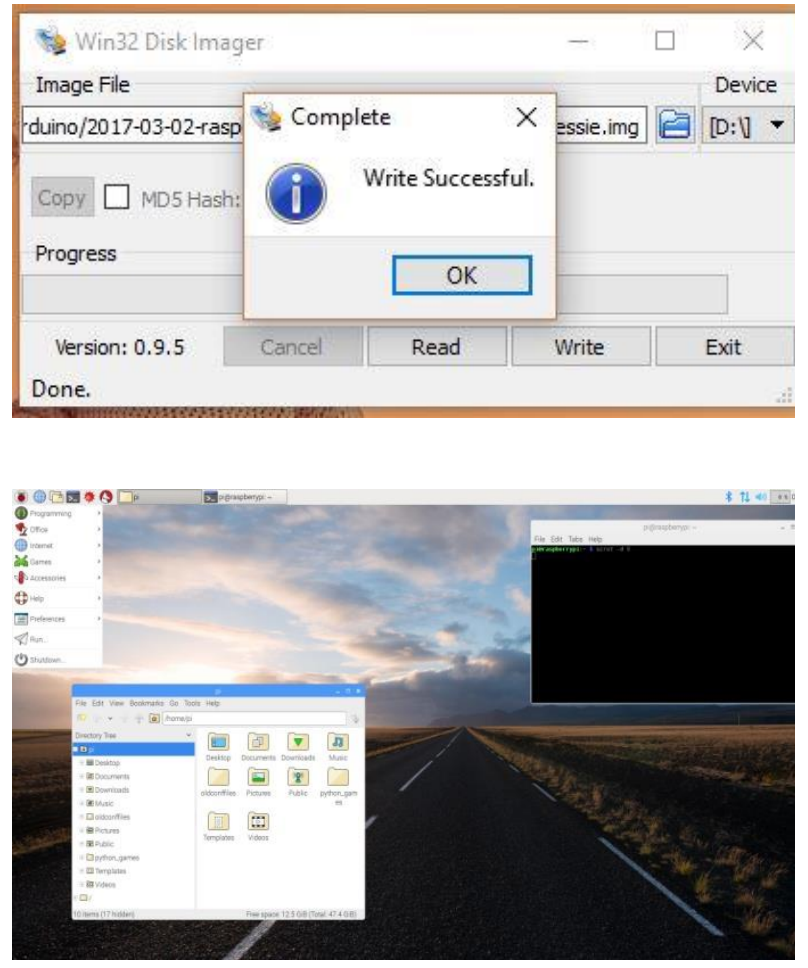

Fig 5: Homepage of the Raspbian OS.

\subsection{Algorithm used in the system}

In our system for image processing open computer vision (open $\mathrm{CV}$ ) tool box is used, it is an open source library it can be used directly in my system. The library which is used here has very important role. The algorithm is implemented in this open computer vision(CV)library. This algorithm gives the exact location of the pupil by the following steps.

- Face \& eye detection: The main objective \& motive of this framework is finding the eye pupil location. For that purpose first step is face detection and eye detection. There is so many algorithms such as template matching, Fisher Linear Discriminant, SVM, MRC and Haar Cascade algorithms are used to find the face detection. In this system the Haar cascade [7] algorithm is used directly. The night vision camera perceives the eye and face of the user exclusively, once it will distinguish the framework found the area of the eye and denoted the eye locale.

- Color Conversion: Once the detection of eye process is finished the size of the color images are high compare to the gray scale images. To process the color images in this system it will take more time. In this system our concentration is only on eye part. Basically the color of the eye part is black and white so in this system the next step is to convert the color image into gray scale image it will help to reduce the system delay time.

- Thresholding and edge detection: In image processing, computer vision and Digital image processing there are so many edge disclosure approaches such as Prewitt, Robert, Canny edge detection, Sobel. Out of these canny edges detection is popular in recent days. For finding the soft edges of the eye the canny edge detection and corner detection algorithms are used. Proper threshold value is chosen to recognize the location of the eye part and pupil part in the form of rectangle or circle in a image.

- Hough transform: In 1962 Paul Hough introduced Hough transform algorithm. The objective of this algorithm is to detect the features in digitalized images of particular shapes like circles or lines [13]. This Hough transform is relatively not affected by image noise; this is the main leverage of this algorithm. It is used for most of the computer vision problems for finding the object boundaries. Night vision Camera catches the pictures consistently. After edge location and thresholding of a picture we are applying the hough circle transform strategy to plot a sphere on the eye pupil.
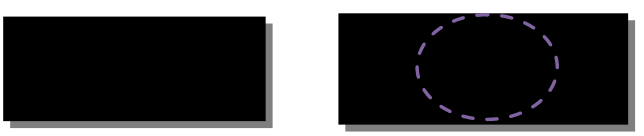

Fig 6: Hough transform output of an image

- Eye motion tracking: Eye tracking [11] method is used to detect the individuals eye movements. The movement detection is done with a very basic principle. We collect the feature points for both left and right eyes \& left and right pupil movement and store it. After that we take the difference in pixels of the left and right pupil position in the current image from the previous image. In each image the difference is calculated, and if this difference above the threshold in any direction right, left indicating the wheel chair directs to left movement or right movement. If the difference is not above the threshold, then we can say that no pupil movement has occurred the wheel chair directs to forward direction.

\section{Results}

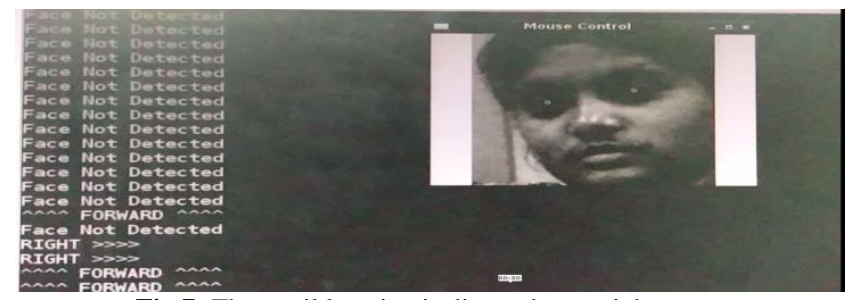

Fig 7: The pupil location is directed to straight 


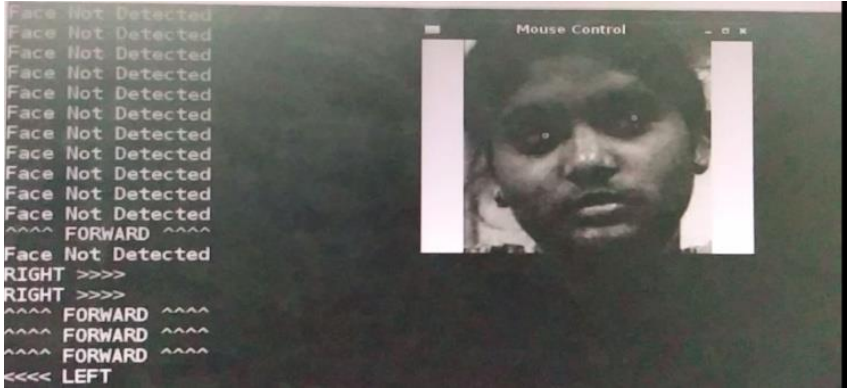

Fig 8: The pupil location is directed to LEFT

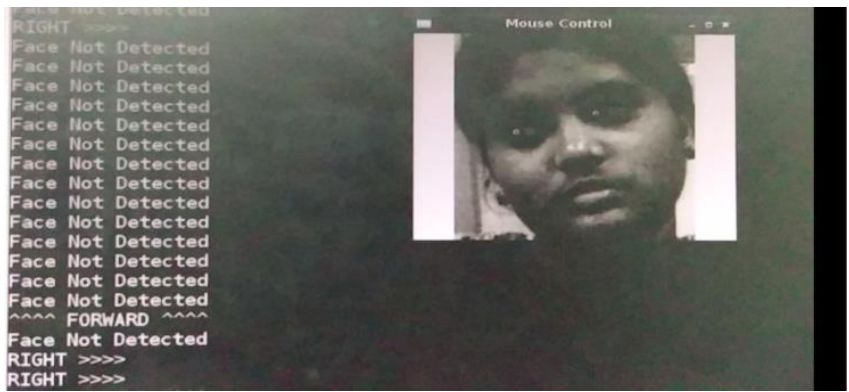

Fig 9: The pupil location is directed to RIGHT
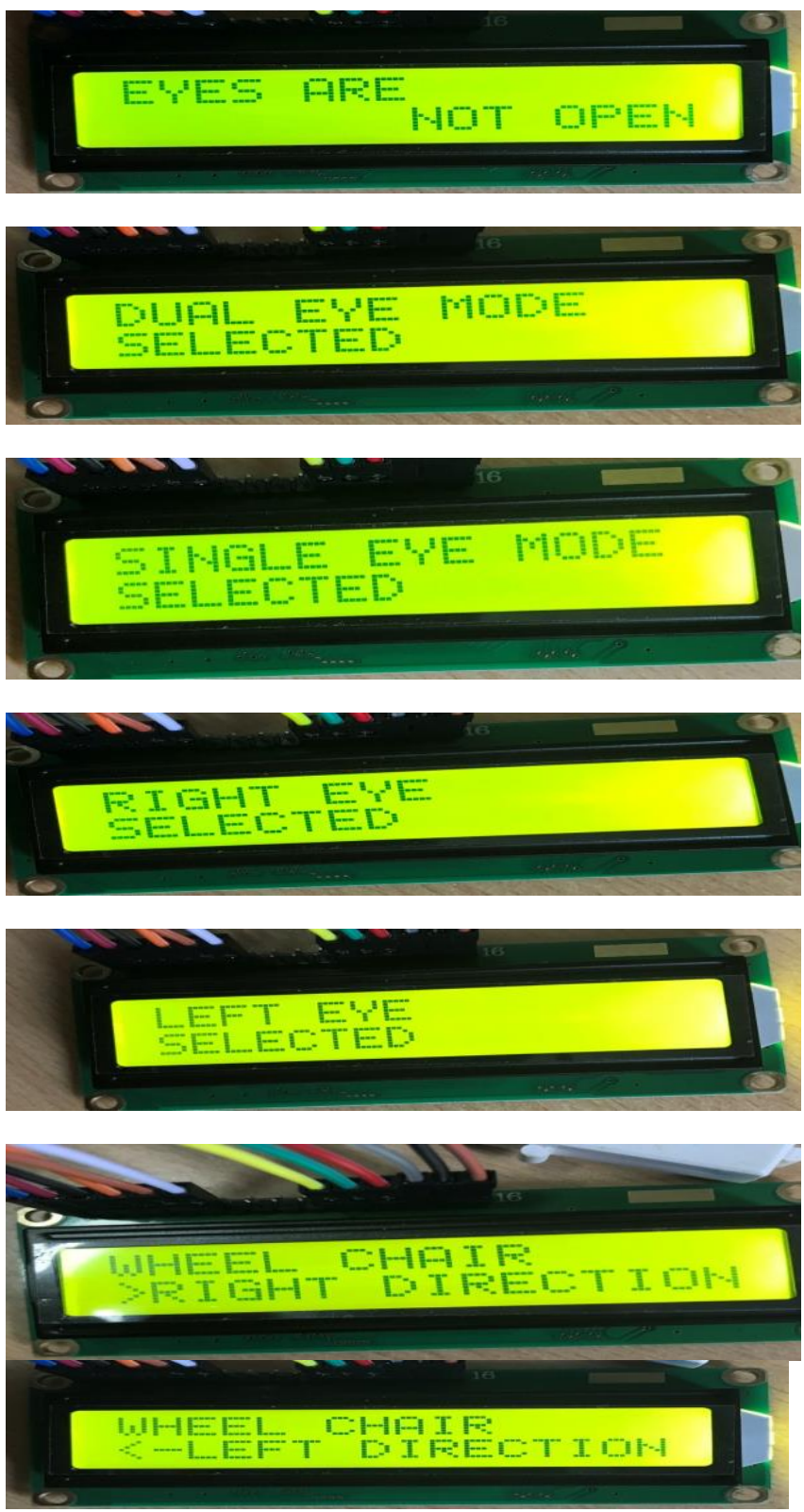
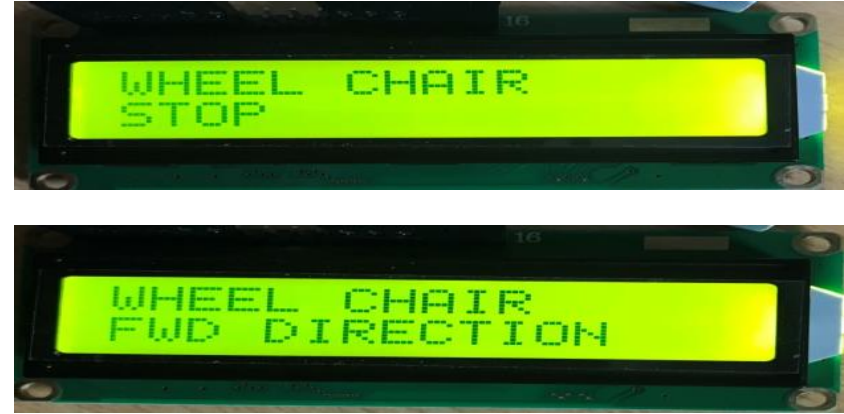

Fig 10: LCD display outputs

\section{Conclusion}

This paper is very helpful for physically handicapped persons, Spinal card injury persons and movement disability persons. This is the on e solution to make their life individual. This system works in all lighting conditions and it works in internal and external environments also. The entire process it takes only 4 seconds and the GPS, GSM modules are used for tracking the whell chair in case of emergency. And this system mainly concentrate on squint eye pupil. It will help full for both squint eye as well as pupil having proper eyes. This system is prototype module for in case real chair system the motor capacity and drivers capacity should be high.

\section{References}

[1] Yash Pathak, Samir Akhare, And Vishal Lambe- Iris Movement Tracking By Morphological Operations For Direction Control,. September 2012.

[2] Sarangi, P., Grassi, V., Kumar, V., Okamoto, J.:॥ Integrating Human Input With Autonomous Behaviours On An Intelligent Wheelchair Platforml, Journal Of Ieee Intelligent System, 22, 2, 33-41, [2007].

[3] Deepak Kumar Lodhi1, Prakshi Vats1, Addala Varun1, Prashant Solanki1, Ritakshi Gupta1, Manoj Kumar Pandey2, Rajat Butola2. Smart Electronic Wheelchair Using Arduino And Bluetooth Module In International Journal Of Computer Science And Mobile Computing, Vol.5 Issue.5, May- 2016, Pg. 433-438

[4] Archibald Danquah-Amoah1 , Daniel Amiteye2 Bassey, Glory Basiono3, Edem, Deborah Okon4 , Alwell Brown Nyingiere5," Assistive Robotic Wheelchair Using Voice And Bluetooth Control" In International Journal Of Scientific Research Engineering \& Technology (Ijsret) Volume 2 Issue 9 Pp 553-559 December 2013.

[5] Matt Bailey, Et. Al, —Development Of Vision Based Navigation For A Robotic Wheelchairl, In Proceedings Of 2007 Ieee 10th International Conference On Rehabilitation Robotics.

[6] Autonomous Camera Based Eye Controlled Wheelchair System Using Raspberry-Pi, Shyam Narayan Patel, Ieee Sponsored 2nd International Conference, 2015.

[7] Shafi. M, Chung. P. W. H:llA Hybrid Method For Eyes Detection In Facial Imagesll, International Journal Of Electrical, Computer, And Systems Engineering, 231-236, [2009].

[8] Luong D. T, Lee H. C, Park K. R, Lee H. K, Park M. S, Cha J (2010) A System For Controlling Iptv At A Distance Using Gaze Tracking. Proc. Ieee Summer Conference. 33: 37-40.

[9] M.Wu, J.-H. She, G.-X. Zeng, And Y. Ohyama, -Internet-Based Teaching And Experiment System For Control Engineering Course, Ieee Trans. Ind. Electron., Vol. 55, No. 6, Pp. 2386-2396, Jun. 2008.

[10] D. Purwanto, R. Mardiyanto, K. Arai: \| Electric Wheelchair Control With Gaze Direction And Eye Blinkingl, Proceedings Of The Fourteenth International Symposium On Artificial Life And Robotics, Gs21-5, B-Con Plaza, Beppu, [2008].

[11] Cho C. W, Lee J. W, Shin K. Y, Lee E. C, Park K. R, Lee H, Cha J (2012) Gaze Detection By Wearable Eye Tracking And Nir LedBased Head- Tracking Device Based On Svr. Etri Journal 34: 542$552 .$.

[12] Autonomous Camera Based Eye Controlled Wheelchair System Using Raspberry-Pi, Shyam Narayan Patel, Ieee Sponsored 2nd International Conference, 2015.

[13] Wojciech Wojcikiewicz, "Hough Transform, Line Detection In Robot Soccer”, Coursework For Image Processing. 14th March 2008. 
[14] Eye Controlled Wheelchair, Sandesh Pai, Sagar Ayare, Romil Kapadia, And October-2012. - Iris Movement Tracking By Morphological Operations For Direction Controll, Yash Pathak, Samir Akhare, And Vishal Lambe. September 2012.

[15] Kumar, R.; Kumar, H., "Availability And Handling Of Data Received Through Gps Device: In Tracking A Vehicle," Advance Computing Conference (Iacc), 2014 Ieee International, Vol., No. Pp.245, 249, 21 - 22 Feb. 2014.

[16] Seokju Lee; Tewolde, G.; Jaerock Kwon, "Design And Implementation Of Vehicle Tracking System Using Gps/Gsm/Gprs Technology And Smartphone Application," Internet Of Things (WfIot), 2014 Ieee World Forum On , Vol., No., Pp.353,358, 6-8 March 2014.

[17] Madhav, B.T.P., Mohan Reddy, S.S., Sharma, N., Ravindranath Chowdary, J., Pavithra, B.R., Kishore, K.N.V.S., Sriram, G. And Sachin Kumar, B., 2013. Performance Characterization Of Radial Stub Microstrip Bow-Tie Antenna. International Journal Of Engineering And Technology, 5(2), Pp. 760-764.

[18] Kishore, P.V.V., Kishore, S.R.C. And Prasad, M.V.D., 2013 Conglomeration Of Hand Shapes And Texture Information For Recognizing Gestures Of Indian Sign Language Using Feed Forward Neural Networks. International Journal Of Engineering And Technology, 5(5), Pp. 3742-3756.

[19] Kishore, P.V.V., Sastry, A.S.C.S. And Kartheek, A., 2014. VisualVerbal Machine Interpreter For Sign Language Recognition Under Versatile Video Backgrounds, 1st International Conference On Networks And Soft Computing, Icnsc 2014 - Proceedings 2014, Pp. 135-140.

[20] Kishore, P.V.V., Prasad, M.V.D., Prasad, C.R. And Rahul, R., 2015 4-Camera Model For Sign Language Recognition Using Elliptical Fourier Descriptors And Ann, International Conference On Signal Processing And Communication Engineering Systems - Proceedings Of Spaces 2015, In Association With Ieee 2015, Pp. 34-38.

[21] Vudatha, C.P., Nalliboena, S., Jammalamadaka, S.K.R., Duvvuri, B.K.K., Reddy, L.S.S., Automated Generation Of Test Cases From Output Domain Of An Embedded System Using Genetic Algorithms, Icect 2011 - 2011 3rd International Conference On Electronics Computer 5,5941989, Pp. 216-220

[22] Sastry, J.K.R., Ganesh, J.V., Bhanu, J.S., I2c Based Networking For Implementing Heterogeneous Microcontroller Based Distributed Embedded Systems, Indian Journal Of Science And Technology, Volume 8, Issue 15, 2015 